\title{
Optimal control strategy to reduce the temporal wavefront error in AO systems
}

\author{
N.J. Doelman ${ }^{* a}$, K.J.G. Hinnen ${ }^{b}$, F.J.G. Stoffelen ${ }^{\mathrm{a}}$, M.H. Verhaegen ${ }^{\mathrm{b}}$ \\ ${ }^{a}$ TNO TPD, Stieltjesweg 1, P.O. Box 155, 2600 AD, Delft, The Netherlands; \\ ${ }^{b}$ Delft Center for Systems and Control, Mekelweg 2, 2628 CD, Delft, The Netherlands
}

\begin{abstract}
An Adaptive Optics (AO) system for astronomy is analysed from a control point of view. The focus is put on the temporal error. The AO controller is identified as a feedback regulator system, operating in closed-loop with the aim of rejecting wavefront disturbances. Limitations on the performance of feedback regulator systems are discussed. The concept of optimal control is proposed to minimise the temporal error. The issue of closed-loop feedback controller design is made transparent by using the principle of Internal Model Control. The central issue in reducing the temporal wavefront is the design of a feedforward prediction filter. In three separate tests - a numerical simulation example, measured data from an AO test bench and open-loop telescope data - the advantage of optimal control over the common approach of integral control is demonstrated. Optimal control of the temporal error yields a smaller temporal error, enables a longer integration time in the wavefront sensor, or the use of fainter natural guide stars.
\end{abstract}

Keywords: adaptive optics, optimal control, temporal error, turbulence prediction, telescope data

\section{INTRODUCTION}

In analysing the performance of adaptive optics systems it is common to distinguish between a number of main error sources. These error sources are known as: anisoplanatic errors, wavefront measurement error, wavefront fitting error, temporal errors and tilt related errors. In this paper we will concentrate on the temporal error only. In particular, the design of the control system to reduce the temporal error will be the central issue. Characteristics of other AO system components, like the wavefront sensor, the deformable mirror, the reference sources and the science object are considered as given and will not be included in the control design study.

The temporal error is generally one of the main error sources limiting the achievable image quality in current AO systems. For the Keck Observatory ${ }^{2}$ e.g. the temporal error is mentioned as one of the three major error sources.

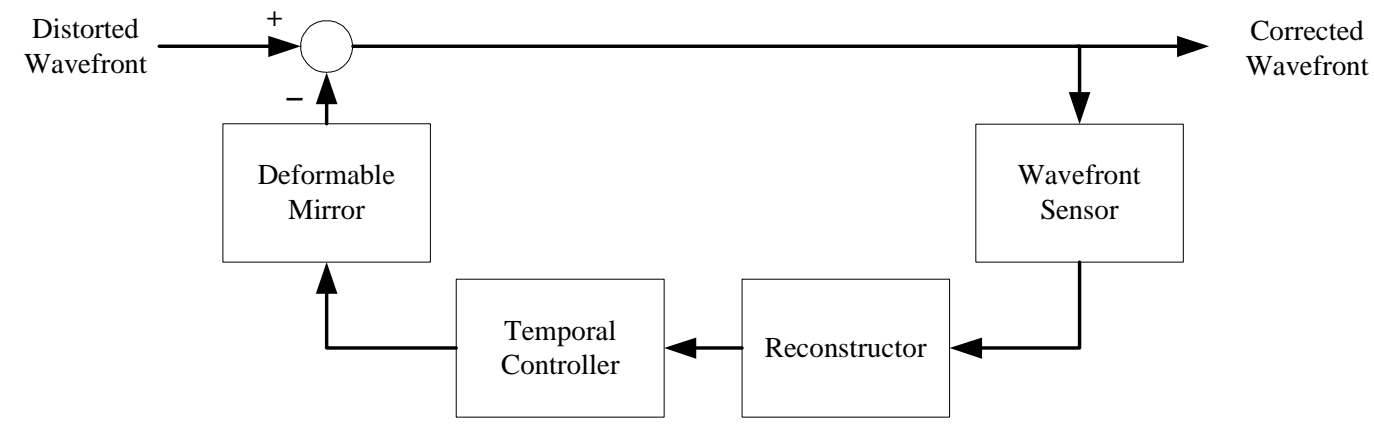

Fig. 1. General AO system block diagram.

A general block diagram of an AO system is depicted in Fig. 1. An AO system of this type is incapable of reacting instantaneously to wavefront disturbances at the very moment of occurrence. Any time delay between measurement of the wavefront and actual correction of the wavefront leads to a temporal error. The two main causes for a delayed response are:

1. The finite bandwidth of the wavefront correction link (wavefront sensor - controller - deformable mirror);

2. A pure time delay in the wavefront correction link.

The finite bandwidth may originate from limitations in the wavefront sensor (e.g. the frame rate of the photon detector, limitations in the dynamic response of the deformable mirror and the finite sampling rate of the (digital) controller). Pure

*doelman@tpd.tno.nl; phone +31 15269 2410; fax +31 15269 2111; www.tpd.tno.nl/smartsite215.html 
time delays may be due to analogue to digital conversion, digital to analogue conversion and computation time in the processor. The effect of these system limitations on the image quality depends on the dynamics of the atmospheric turbulence. The faster the turbulence dynamics, the more serious the influence of a limited bandwidth or a time delay will be on the resultant image quality.

\section{THE TEMPORAL ERROR IN AN AO SYSTEM; CONTROL LIMITATIONS}

From a control point of view an AO system is a multiple-input multiple-output regulator system. The primary task of a regulator system is to keep a designated array of outputs at predetermined values despite the presence of disturbances. In an AO system the disturbance at any output has a stochastic nature and its power spectral density follows basic laws. Usually, the power spectrum of the wavefront phase disturbance is referred to as a Kolmogorov-type of spectrum (it falls of with frequency $f^{-8 / 3}$ ), although strictly speaking this does not hold for the entire spectral range.

Since no advance information of the disturbance is measurable or available, the regulator system has a feedback control architecture. This means that deviations of the outputs from the desired values are passed to the controller after they have occurred at the outputs. Re-organizing the block diagram of Fig. 1 yields a common feedback control configuration as shown in Fig.2. The signals in the diagram represent multi-variable data vectors.

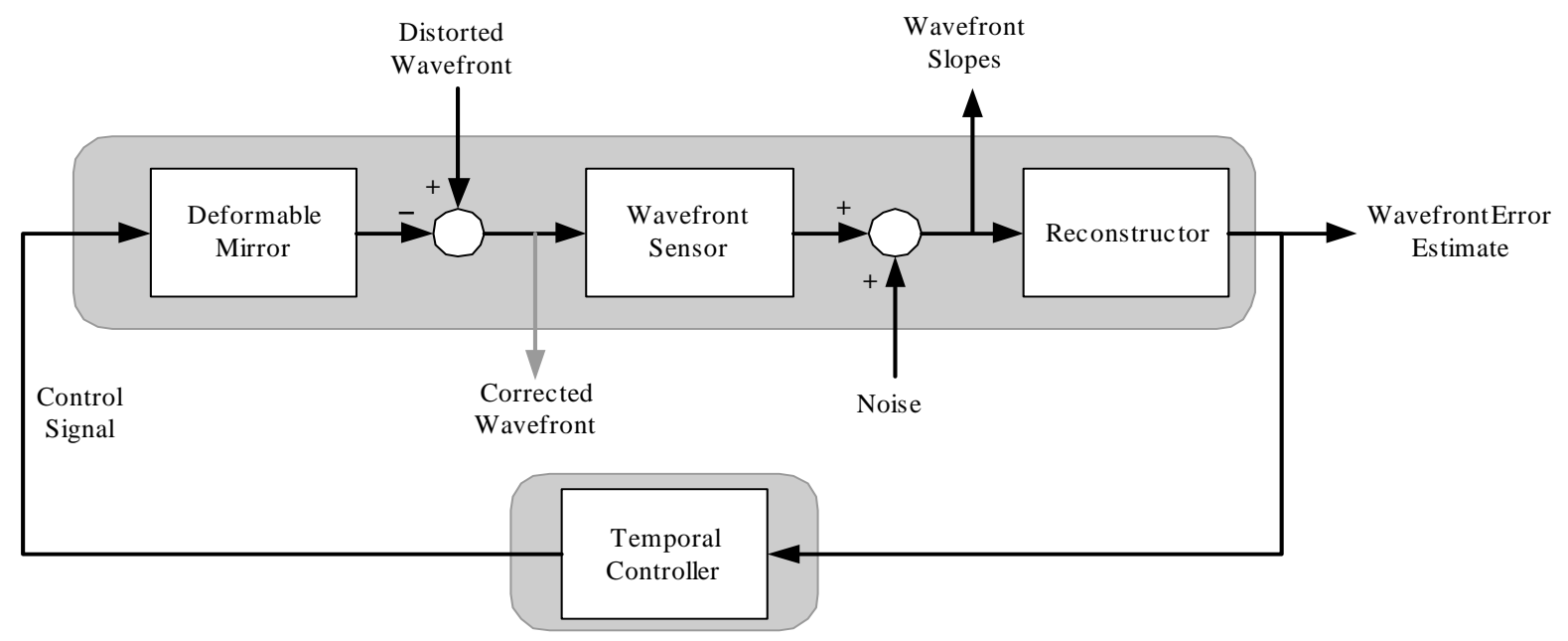

Fig. 2. AO system diagram with a clear distinction between plant and controller.

The design problem is to find an optimal controller that minimises the wavefront disturbance as it appears in the observed image, whereas the wavefront sensor (typically a Shack-Hartmann sensor) provides spatially sampled information of the gradient of the wavefront. In addition to this sensor inherent noise is introduced to the system. Furthermore, stable closed-loop behaviour is a must and robustness of performance a prerequisite.

All the above together constitutes a challenging control design problem. Unfortunately, it is beyond the scope of this paper to address it completely. We will focus on the control of the temporal error. The wavefront reconstruction method will not be considered here. It is assumed that the wavefront reconstruction algorithm adequately deals with the properties of the wavefront sensor and delivers an accurate estimate of the wavefront disturbance. The estimated wavefront disturbance then is the quantity to be optimised by the controller. The structure of this control system is shown in Fig. 3. Note that in Fig. 3 the series connection of deformable mirror, optical transfer path, wavefront sensor and wavefront reconstruction is considered as a single system. In the control community this system is usually denoted as the plant; represented by $\mathbf{G}$ in the diagram. The open-loop series connection of plant and control system $\mathbf{C} \cdot \mathbf{G}$ is often denoted as the loop transfer function. The disturbance at the output of the system now is the wavefront distortion as it is estimated by the wavefront reconstruction algorithm. It obviously differs from the true wavefront disturbance, but it represents the information of the wavefront distortion as it is passed to the controller. In optimising the controller we could choose to minimise this disturbance or some filtered version of it. The filter would be being chosen such to correct for the unwanted transfer characteristics of the wavefront sensor and reconstruction algorithm. 


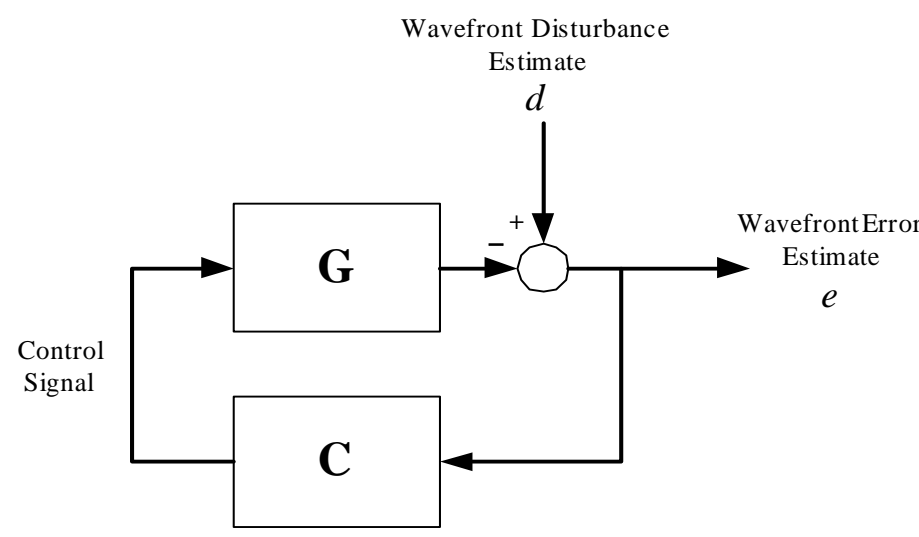

Fig. 3. Diagram of an AO system as a feedback regulator system.

In single-input, single-output form the disturbance rejection of this feedback control system as a function of frequency can be written as:

$$
E(j \omega)=S(j \omega) D(j \omega)
$$

where $E(j \omega)$ and $D(j \omega)$ are the Fourier transforms of the (estimated) wavefront error and the (estimated) wavefront disturbance respectively, both signals as a function of the radial frequency $\omega$. The function $S$ is known as the sensitivity function. It describes the disturbance rejection ratio for the feedback control system as a function of $\omega$. The performance of the feedback controller in rejecting stochastic disturbances is limited by some fundamental rules; see for instance ${ }^{3,4}$. These limitations are imposed by the constraint of causal action and the plant pole-zero configuration.

A well-known limitation is given by Bode's integral constraint. For a well-posed system it can be formulated as:

$$
\int_{0}^{\infty} \log |S(j \omega)| d \omega=\gamma
$$

where $\gamma \geq 0$, determined by the poles and the pole excess of the loop transfer function $\mathbf{C}(j \omega) \mathbf{G}(j \omega)$. The minimum value of $\gamma$ and thus of Bode's integral is zero. In simple words this means that the sensitivity function cannot be made small for all frequencies. If one achieves disturbance rejection in a certain frequency range, then one would achieve disturbance amplification in another frequency range. The performance limitations get more stringent in the case the plant contains unstable poles. Bandwidth limitations occur when the plant contains unstable zeros or time delays. A time delay in particular sets a bound to the maximum bandwidth of disturbance rejection. For multiple-input multiple-output similar restrictions apply.

Generally for an AO system, properties like unstable zeros or unstable poles are determined by the dynamical behaviour of the deformable mirror (DM) and the wavefront sensor (WFS). In the line of the paper these system aspects are regarded as invariable. The same holds for the time delay, although it is influenced by the selection of the controller sample rate, the processing time and also the integration time in the wavefront sensor. The main question here is however, how to get the best out of the AO control system given the fact that there is a time delay and some fundamental performance limitations that come along with it.

With the laws of control theory in mind, two design guidelines emerge:

1. If the disturbance rejection ratio over frequency has to obey strict rules, it would be most effective to fully tune the feedback control system to the characteristics of the atmospheric turbulence. That is: get disturbance attenuation at those frequencies where it is largest and allow amplification where it is least detrimental. This implies that in control design a model of the disturbance is estimated in some explicit or implicit way. It could also imply that over a period of time this model needs to be adapted to the time-varying characteristics of the atmospheric turbulence. Adaptive or non-adaptive, the controller is designed on the basis of the disturbance model properties.

2. A way to escape from the performance limits for feedback control systems would be to acquire time-advanced information of the disturbance. This is a common approach in control in the case that the disturbance is a propagating phenomenon. It enables a feedforward control link and this is the way to beat the plant time-delay. For AO systems additional sensors could provide early disturbance characteristics based on meteorological data and a physical model or wavefront measurements beyond the aperture. The most obvious configuration is inspired by the Taylor frozen flow hypothesis. Wavefront measurements at subapertures on the windward side of the telescope contain information of the future wavefront distortion in subapertures on the lee side of the telescope. Even if Taylor's hypothesis only partially holds, an additional feedforward connection based on this principle may improve the AO system performance. Most improvement is to be expected for AO systems in which the wavefront sensors distinguish between several turbulence layers (Multi Conjugate AO). 


\section{THE TEMPORAL ERROR IN AN AO SYSTEM; CURRENT APPROACHES}

In current research for $\mathrm{AO}$ the issue of wavefront reconstruction draws a lot of attention ${ }^{5}$. Proper estimation of the wavefront distortion in the presence of measurement noise is crucial to enable the calculation of deformable mirror actuator commands. This has lead to minimum-variance type or maximum-likelihood type of wavefront estimators. Here, the Kolmogorov-type statistics of the wavefront disturbance are explicitly taken into account. Based on the Wallner decomposition ${ }^{6}$ the DM actuator commands follow after a least-squares fit of the estimated wavefront phase to the actuator inputs.

To make this more explicit, consider the common WFS measurement model:

$$
s=A \varphi+\eta
$$

where $s$ is the vector of wavefront slopes as measured by the wavefront sensor, the geometry matrix $A$ specifies how subaperture slopes are related to nearby wavefront phases and $n$ represents measurement noise.

Furthermore, let the DM be modelled by the matrix equation

$$
\varphi_{D M}=B u
$$

where $\varphi_{D M}$ is the deformable mirror figure as a result of the actuator input or control signal $u$. The matrix $B$ is often referred to as the poke matrix. The minimum variance reconstruction solution is equivalent to the Wiener estimate:

$$
\hat{\varphi}=\arg \min _{\varphi}\left\{\|A \varphi-s\|_{2}^{2}+\sigma_{\eta}^{2} \varphi^{T} C_{\varphi}^{-1} \varphi\right\}
$$

where $C_{\varphi}$ is the spatial covariance matrix of the uncontrolled wavefront (Kolmogorov type) and $\sigma_{\eta}$ is the variance of the (white) measurement noise. The DM actuator command vector follows subsequently as:

$$
u=\left[B^{T} B\right]^{-1} B^{T} \hat{\varphi}
$$

Since both fitting and estimation involve matrix inversion steps, efficient and fast converging algorithms are being developed to enable attractive implementation for large-scale AO systems ${ }^{5}$.

Although, wavefront reconstruction has been left out of the discussion on control performance in Section 2, the common wavefront reconstruction approach as described above reveals an alternative look on the control issue:

- The DM actuator signals are regarded as servo commands to the actuators. With a servo task, the controller needs to react such that the deformable mirror figure changes according to the commands. A servo control approach differs from the disturbance rejection approach discussed in the previous section.

- With the servo control approach the presence of closed-loop operation is in fact neglected. The response of the DM actuator signals on the wavefront slopes $s$ is not taken into account in the calculation of control signal $u$. Fitting the wavefront estimate on the DM actuator input is performed with an open-loop DM transfer function model (the poke matrix). That is in contrast with the previous section where it was concluded that controller task is to reject the disturbance in a closed-loop, feedback setting. Also, the Wiener estimate is based on the open-loop wavefront disturbance covariance properties $C_{\varphi}$.

- The dynamics of the DM structure and actuators are often disregarded. The fitting operator only deals with the spatial response of each DM actuator and does not account for the temporal response.

As a result of this the closed-loop stability in this common control approach is ill-defined. Regularly, a temporal control action is inserted in between the DM actuator commands and the actuators. In designing this temporal controller it is generally assumed that the control loop can be split up into independent single-input single-output loops operating in parallel. These single-channel loops could either have a zonal basis or modal basis.

The individual control loops usually have a predefined input-output structure, which has its roots in classical control theory. It is common to denote this control approach in discrete-time. Typically, a single-channel control action has a zdomain transfer function:

$$
C_{\text {int }}\left(z^{-1}\right)=\frac{\beta}{1-\alpha z^{-1}}
$$


Here, the parameter $\alpha$ equals unity or is close to unity, which implies that the control law represents a (leaky) integrator. With the selection of an integrating controller any bias introduced by the open-loop approach is automatically eliminated. Since the wavefront estimate (eq. 5) in closed-loop actually stands for an estimate of the residual wavefront, the DM actuator command actually is an incremental command. The deformable mirror should not forget its previous action, which is taken care of by the integrator. With respect to closed-loop stability the loop-gain parameter $\beta$ can be tuned. In a paper by $\mathrm{Wild}^{7}$ a Lyapunov stability study is described, presenting suitable ranges for $\beta$ as a function of the wavefront estimation method.

More sophisticated temporal controllers have also been described, like a combined integrating and Smith predictor control law ${ }^{8}$. Smith predictors are usually implemented to compensate the effect a time-delay has on the closed-loop behaviour.

Comparing the common approach with the remarks in Section 2, it should be mentioned that the common approach with respect to temporal control is a heuristic one. With PID-type of controllers the closed-loop is stabilized and the potential detrimental effect of loop delay is circumvented by a Smith predictor configuration. No specific knowledge of the Kolmogorov disturbance is utilized to design the temporal controller for optimal rejection or sensitivity within Bode's integral constraint. The same holds for the dynamics of the plant. Although, the dynamical behaviour of DM and WFS may be fast and well-damped, it might be useful to include these in the control design.

\section{STRATEGY TO REALISE OPTIMAL CONTROL}

In this section we will give an outline of a control approach that aims at minimisation of the temporal error. The concept of 'optimality' or 'minimisation' is always related to a criterion function. The fundamental criterion function is the time and space-averaged square of the wavefront phase error $e$. Mathematically, this can be expressed as

$$
J_{1}=\lim _{T \rightarrow \infty} \frac{1}{T} \frac{1}{S_{a}} \int_{0}^{T} \int_{S_{a}}\left(e\left(r_{s}, t\right)\right)^{2} d r_{s} d t
$$

in which $S_{a}$ is the telescope aperture area and the wavefront phase error $e$ a function of spatial co-ordinates $r_{s} \in S_{a}$ and time $t$. Minimisation of the average squared wavefront phase error is closely related to maximization of the Strehl ratio through Marechal's approximation. In practice, both spatial and temporal AO system dynamics can be regarded as bandlimited which allows sampling in both spatial and temporal sense. The corresponding criterion function is

$$
J_{2}=\lim _{N \rightarrow \infty} \frac{1}{N+1} \frac{1}{N_{s}} \sum_{n=0}^{N} \sum_{i, j} e_{i j}[n]^{2}
$$

in which $N_{s}$ is the number of spatial points of wavefront evaluation and $e_{i j}[n]$ denotes the wavefront phase error at sampling instant $n$ at aperture position $(i, j)$.

The general control design problem is to determine a controller that minimises the averaged square wavefront error (eq. 8 or eq. 9) with the additional constraints that:

- the wavefront sensor (typically Shack-Hartmann type) provides the controller with an estimate of the gradient of the wavefront;

- the wavefront sensor measurements are contaminated with noise;

- the controller is to operate in closed-loop;

- the controller is to drive the actuators of a deformable mirror with specific dynamic behaviour.

\subsection{Identification of the central control issue}

For convenience the controller is restricted to be linear and time-invariant. The latter property would exclude the possibility to track changes in disturbance and plant dynamics. Such an adaptive control strategy could prove very useful in practice. Adaptive control is beyond the scope of this paper however and therefore the plant and the turbulence disturbance are assumed to be time-invariant as well.

In the remainder of this section the optimal control design problem will be dissected in a few steps in order to focus on the central issue. It will turn out that minimisation of the temporal error is closely related to optimal prediction of the wavefront disturbance. 
a) accurate wavefront estimation. - As shown in Section 3 it is common in AO systems to accept the outcome of the wavefront reconstruction algorithm as the best estimate of the actual wavefront phase. Therefore, the estimate is put forward as the DM servo command signal, representing the desired mirror figure to cancel the wavefront distortion. So the effective criterion function for control design has become the average squared estimated wavefront error. With this step we have returned to the control configuration of Fig. 3.

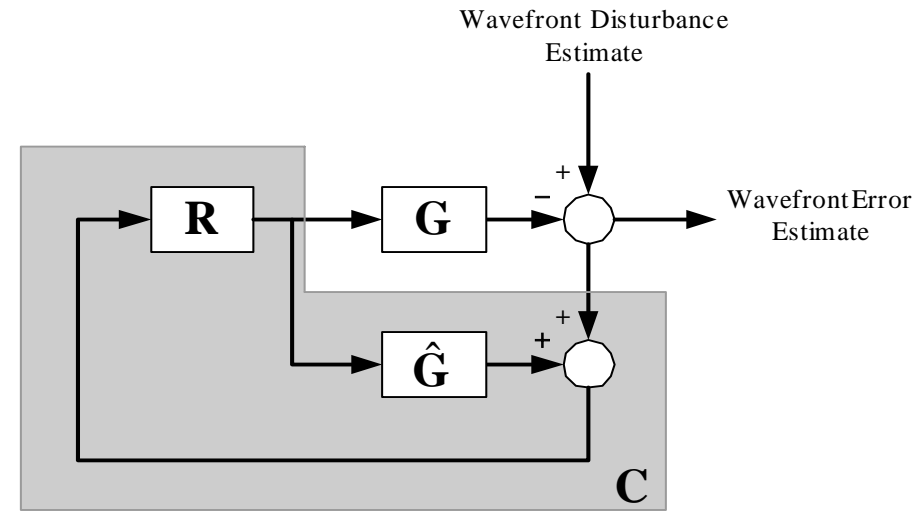

Fig. 4. Block diagram of the feedback control system with an internal model control implementation. b) accurate plant model - The closed-loop setting of the AO controller (see Fig. 3) complicates the control design. First, the demand of closed-loop stability emerges alongside the requirement of minimal wavefront error. Second, the controller enters in the expression for the wavefront error in a non-linear way. Using socalled Internal Model Control the closed-loop design problem can be disentangled without changing the performance limitations of the system. In Fig. 3 the plant $\mathbf{G}$ was introduced as the series connection of deformable mirror, optical transfer path, wavefront sensor and wavefront reconstruction. Assume a model $\hat{\mathbf{G}}$ of this plant has been acquired. Both the plant and the plant model are open-loop stable. Now consider the following controller structure of Fig. 4.

This control configuration is known as Internal Model Control ${ }^{9}$. The relation between the original controller $\mathbf{C}$ and the new controller $\mathbf{R}$ is given by:

$$
\mathbf{C}=[\mathbf{I}-\mathbf{R} \hat{\mathbf{G}}]^{-1} \mathbf{R}
$$

It can be demonstrated that any stabilizing controller $\mathbf{C}$ can be put in the form of Fig. 4 and eq. (10) if $\mathbf{R}$ is open-loop stable. So the controller $\mathbf{R}$ can be considered the parameterisation of all stabilizing controllers. Still, the optimum performance of the systems in Fig. 3 and Fig. 4 are equivalent. Next, assume that the plant model is perfect; $\hat{\mathbf{G}}=\mathbf{G}$. Then, the control configuration simplifies to the one shown in Fig. 5.

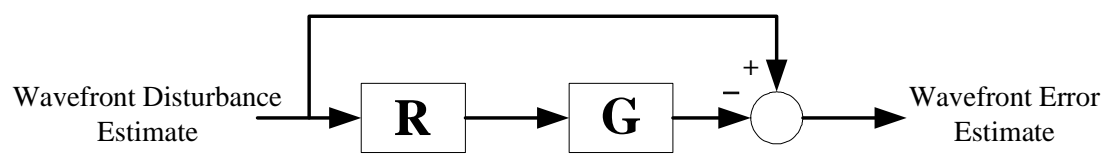

Fig. 5. Equivalent block diagram for the internal model control arrangement in the case of a perfect plant model.

The control setting of Fig. 5 corresponds to open-loop control. It actually resembles a feedforward control arrangement. It should be noted however, that the disturbance signal at the input of the controller $\mathbf{R}$ does not contain time-advanced information, as it passes to the system output without any delay.

Because $\mathbf{R}$ still represents the class of all stabilizing controllers, the optimal $\mathbf{R}$ yields the same performance as the optimal $\mathbf{C}$. The demand of stability can be resolved easily. The control system is stable if the controller $\mathbf{R}$ is stable.

The internal model principle has been applied here to facilitate the theoretical analysis of optimal control. In practice the internal model principle is also quite valuable. In that case, care must be taken with respect to the plant model error $(\mathbf{G}-\hat{\mathbf{G}})$.

c) ideal plant dynamics compensation - As a final step in the dissection of the control design we will assume that the spatial and temporal plant response properties can be perfectly compensated - except for the time delay. Spatial compensation is common in AO systems, since this is the underlying idea of inverting the poke matrix. Obviously, with this assumption we would ignore the 'fitting error', but as stated in the beginning the focus lies on the temporal error only. Ignoring the fitting error and assuming ideal inversion of the poke matrix, the outputs of the plant can be controlled individually.

Compensation of the temporal behaviour of $\mathbf{G}$ is generally not feasible. Although, the plant is assumed to be stable, the occurrence of unstable transfer zeros would prevent perfect, causal compensation. In our quest for the limitations of 
optimal control, perfect and stable compensation of the plant dynamics is assumed. The reason being that it provides insight into the bounds on performance with optimal control. In practice, this limit will be valid for plants with causal and stable inverses only.

Assuming the possibility of perfect plant compensation (except for the time delay) and ignoring the wavefront fitting error, the control configuration of Fig. 5 can be simplified to the one shown below.

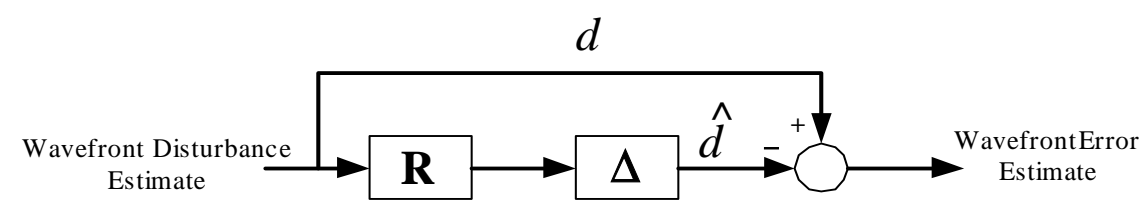

Fig. 6. Block diagram representing the central control design issue.

We now have arrived at the central design issue with respect to minimisation of the temporal error. As is apparent from Fig. 6, in order to reject the wavefront disturbance the controller $\mathbf{R}$ needs to predict the disturbance over a horizon equal to the plant time delay. The underlying criterion function is denoted as

$$
J_{3}=\lim _{N \rightarrow \infty} \frac{1}{N+1} \frac{1}{N_{s}} \sum_{n=0}^{N} \sum_{m}\left(d_{m}[n]-\hat{d}_{m}[n]\right)^{2}
$$

Note that compared to criterion function $J_{2}$, for convenience of notation the sensor points are now re-organized in a vector array with running index $m$, instead of the matrix array with running indices $i, j$.

The disturbance prediction for sensor point $(i, j)$ can be written as

$$
\hat{d}_{m}[n]=\sum_{k} R_{m k} d_{k}\left[n-\Delta_{m k}\right]
$$

in which $\Delta_{m k}$ is the plant time delay for the transfer from actuator channel $k$ to sensor point $m$. The delay $\Delta_{m k}$ has an integer value. The controller $R_{m k}$ is a discrete-time operator, filtering the measured disturbance signal $d_{k}$ such as to obtain an optimal $\Delta_{m k}$ steps ahead prediction of $d_{m}$. The controller $R_{m k}$ can be cast into several model structures, like a statespace form, a lattice filter or an input-output form. In the latter case, the general structure takes the form of an ARMA filter. In terms of the $z$-domain variable this is:

$$
R_{m k}(z)=\frac{\sum_{p=0}^{N_{p}} b_{p} z^{-p}}{1+\sum_{q=1}^{N_{q}} a_{q} z^{-q}}
$$

The control design problem for optimal wavefront disturbance rejection now boils down to finding the optimal values of the prediction filter coefficients $b_{p}$ and $a_{q}$ for each controller $R_{m k}$, such that criterion $J_{3}$ is minimised.

The way to find the optimal prediction filters is not fixed. Depending on the model structure of the controller $R(z)$ several optimisation methods can be selected. One could follow an off-line approach or an on-line approach. Effectively, the optimal solution for $R(z)$ corresponds to the multi-channel, causal Wiener prediction filter.

\subsection{Special choices for the prediction filter}

With the controller structure formulated above, all wavefront disturbance signals contribute to the prediction of the disturbance at each individual sensor channel. In practice, this controller structure might demand too much computational effort. Moreover, it seems unlikely that each sensor signal has a unique component that is correlated with the future disturbance in all other sub-apertures. On physical grounds we can state that the disturbance signals that most likely contain valuable time-advanced information of the disturbance in a specific subaperture are:

- the signals from neighbouring subapertures in the upstream direction of the flow for each turbulence layer; assuming Taylor's frozen flow hypothesis holds.

- the signal from the subaperture itself; the turbulence itself evolves in time and exhibits a certain auto-correlation. 
For these favourite reference sources the prediction operation can be written as:

$$
\hat{d}_{m}[n]=\underbrace{R_{m m} d_{m}\left[n-\Delta_{m m}\right]}_{\text {temporal reference }}+\underbrace{\sum_{l=1}^{N_{l}} R_{m l} d_{l}\left[n-\Delta_{m l}\right]}_{\text {spatial references }}
$$

where the summation over $l$ indicates the subapertures that are positioned between the subaperture in question and the boundary of the aperture in the opposite direction of the flow. For this reason $N_{l}$ is a small subset of the total number of subapertures. The first part of the predictor (eq. 14) relies on the auto-correlation properties of the turbulence and the second part relies on the spatial correlation of the turbulence.

\subsection{Relation to recent advances in $\mathrm{AO}$ control}

The integral controller of Section 3 can be regarded as a special case of the predictive controller described here. In the integral control approach, the current wavefront estimate is seen as the best estimate for future values. So, according to this philosophy neighbouring wavefront estimates or wavefront estimate histories are neglected. The control law would simplify to

$$
\hat{d}_{m}[n]=r_{m m} d_{m}\left[n-\Delta_{m m}\right]
$$

where $r_{m m}$ is just a gain (where it is an operator in eq. (14)). For $\Delta_{\mathrm{mm}}=1$, this open-loop control law is equivalent to the integral controller (eq. 7) operating in closed-loop, under the assumptions made in the beginning of this section. For $\Delta_{\mathrm{mm}}>1$ the open-loop controller (eq. 15) is equivalent to an integral controller with a suitably chosen Smith predictor. This type of control (eq. 15) is sometimes referred to as 'zero-order prediction' ${ }^{1}$. It is well suited if the turbulence disturbance would behave like a random walk phenomenon.

Recently, several researchers have proposed to improve the performance of AO system using predictive control. Mcguire $^{10}$ and co-authors describe an open-loop prediction approach in which the predictors are trained using several recursive algorithms. Tested on simulation data, the predictive approach shows superior performance over the common random walk based control approach.

Dessenne ${ }^{11}$ describes a modal predictive approach. The control system is split up into a set of single-variable servo loops. The controller coefficients are estimated using a recursive least-squares algorithm. The performance obtained is better than achieved by the common integral controller. The authors have also reported sky experiments.

Finally, Page ${ }^{12}$ and Shöck have performed a study of the auto- and cross-correlation properties of WFS data. It can be used to identify individual layers of moving turbulence 'screens' together with the transverse velocity. This in turn can be a valuable input to the design of linear prediction filters.

It should be remarked here that indeed wavefront disturbance prediction is a powerful approach to reduce the temporal error in AO systems. This will be illustrated in the coming sections with several types of experiments. It is however not the complete story of control for AO. In optimal control wavefront prediction takes a prominent place together with closed-loop design, stability, wavefront reconstruction methods, measurement noise propagation, control of the dynamic behaviour of the deformable mirror and the wavefront sensor and robustness towards model uncertainties.

\section{RESULTS ON A SIMULATED TURBULENCE MODEL}

As a first example of the potential of optimal control, a straightforward numerical test has been performed. It concerns a single-input single-output system. So, only the temporal aspects of AO disturbance control are included and all spatial effects are eliminated in this test. Although not realistic for practical AO systems, this example will clearly demonstrate the issue of optimal control of the temporal error.

Consider a discrete-time model of a turbulence-induced wavefront disturbance at a single position in the aperture. In the $z$-domain variable the $5^{\text {th }}$ order model reads:

$$
d=\frac{0.4101-1.2207 z^{-1}+1.1939 z^{-2}-0.3076 z^{-3}-0.1334 z^{-4}+0.0576 z^{-5}}{1-4.5482 z^{-1}+8.2278 z^{-2}-7.3939 z^{-3}+3.2971 z^{-4}-0.5828 z^{-5}} w
$$


Here $d$ is the wavefront disturbance and $w$ is white noise, of which the variance can be tuned to get the desired power. The model has been obtained by matching the model coefficients such that the resultant power spectrum follows the Kolmogorov asymptote $\left(\sim f^{-8 / 3}\right)$. The discrete model sampling frequency is $300 \mathrm{~Hz}$.

Now assume that the plant contains a time delay of 1 sample instant. The random walk approach, or zero order prediction method would then select for the closed-loop controller a pure integrator (eq. 7) with both $\alpha$ and $\beta$ equal to 1 . The corresponding open-loop controller is: $R_{R W}=1$.
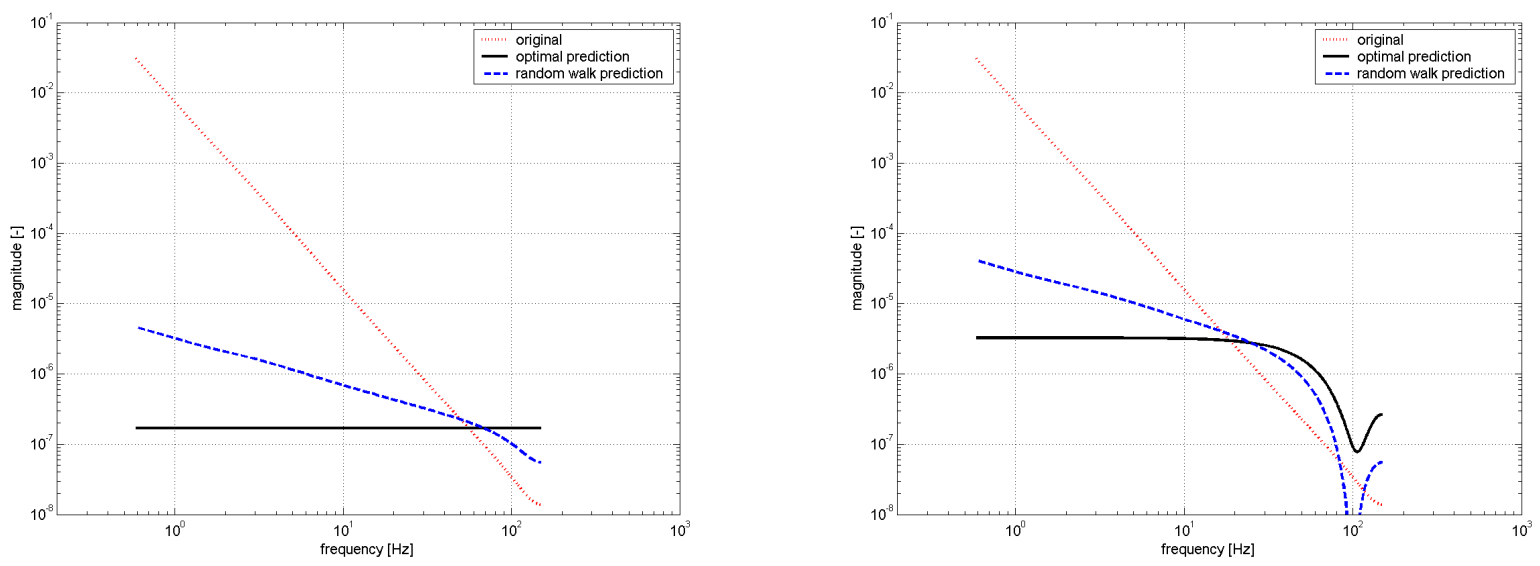

Fig. 7. Comparison of resultant power spectra for optimal prediction and random walk prediction for a delay of one sample (left) and a delay of three samples (right).

The optimal controller for this case equals:

$$
R_{\text {opt }}=\frac{1.5719-5.3170 z^{-1}+6.6440 z^{-2}-3.6223 z^{-3}+0.7234 z^{-4}}{1-2.9763 z^{-1}+2.9108 z^{-2}-0.7499 z^{-3}-0.3252 z^{-4}+0.1405 z^{-5}}
$$

The performance of both controllers is shown in Fig. 7 (left) in terms of power spectra. A distinct difference can be noticed with respect to disturbance rejection. The optimal controller completely whitens the disturbance signal. The random-walk based controller achieves less rejection, especially for the important low frequency band.

For a plant delay of 3 samples a similar experiment can be performed. The disturbance rejection will obviously be less in this case. The random-walk based controller would still take the last wavefront value as best estimate for 3 time steps ahead. In closed-loop this is an integral controller in a Smith predictor configuration. The optimal controller can be found as

$$
R_{\text {opt }}=\frac{2.0448-7.0767 z^{-1}+9.0895 z^{-2}-5.1256 z^{-3}+1.0680 z^{-4}}{1-2.9763 z^{-1}+2.9108 z^{-2}-0.7499 z^{-3}-0.3252 z^{-4}+0.1405 z^{-5}}
$$

Fig. 7 (right) shows the spectral results, which are in agreement with the one sample delay experiment.

\section{RESULTS ON UNCONTROLLED TELESCOPE DATA}

Uncontrolled telescope data from the William Herschel Telescope, situated on La Palma, Canary Islands, have been obtained. The data sets were recorded on June 5 and 8, 1997, in good seeing conditions, 0.5-0.7 arcsec FWHM. The instrument used is the JOSE seeing monitor ${ }^{13}$. Its key features are an $8 \times 8$ Shack-Hartmann array with $0.5 \mathrm{~m}$ subapertures and 2.4 arcsec field of view, imaged onto a $64 \times 64$ pixels CCD, resulting in a pixel scale of 0.27 arcsec/pixel and it is operated at a wavelength of $700 \mathrm{~nm}$. The data sets were taken at a frame rate of $296 \mathrm{~Hz}$ and each set contained 10.000 centroid positions on the CCD with an accuracy of approximately 0.1 pixels. A modal reconstruction method has been used to estimate the phase. This has been done for a grid of $9 \times 9$ phase points, of which 64 are active, matching the Shack-Hartmann sensor used. The mean spatial power spectrum of the resulting phase estimate shows good comparison with the theoretical Kolmogorov model. 
Temporal phase point prediction as described in Section 4 has been compared to the common integral control approach, The predictive control algorithm uses filters with a length of 100 taps; $N_{q}=0$ and $N_{p}=99$ in eq. (13) and includes only the temporal reference, $N_{l}=0$ in eq. (14). Fig. 8 shows the results for one data set taken in the night of June 8,1997 , for a delay of one sample instant. Here the average power spectral density of the spatial aberration is shown for the original (uncontrolled) phase estimate, the integral control approach result and the predictive control result.

The disturbance rejection ratio of the averaged squared error over time and space is $1.1 \cdot 10^{-2}$ for the integral control result and $2.5 \cdot 10^{-3}$ for the predictive control result. These numbers can be translated into an average Strehl ratio. For the original phase estimate the average Strehl ratio is 0.11 , for the integral control result this is 0.56 and for the predictive control result this is 0.85 .

The peaks in the original power spectrum around 50 and $100 \mathrm{~Hz}$ most probably have a non-optical origin and therefore the extra reduction gained here should not be taken into account. However, apart from this, it is clear that predictive control has a significant advantage over the integral control approach. It more or less whitens the disturbance spectrum.

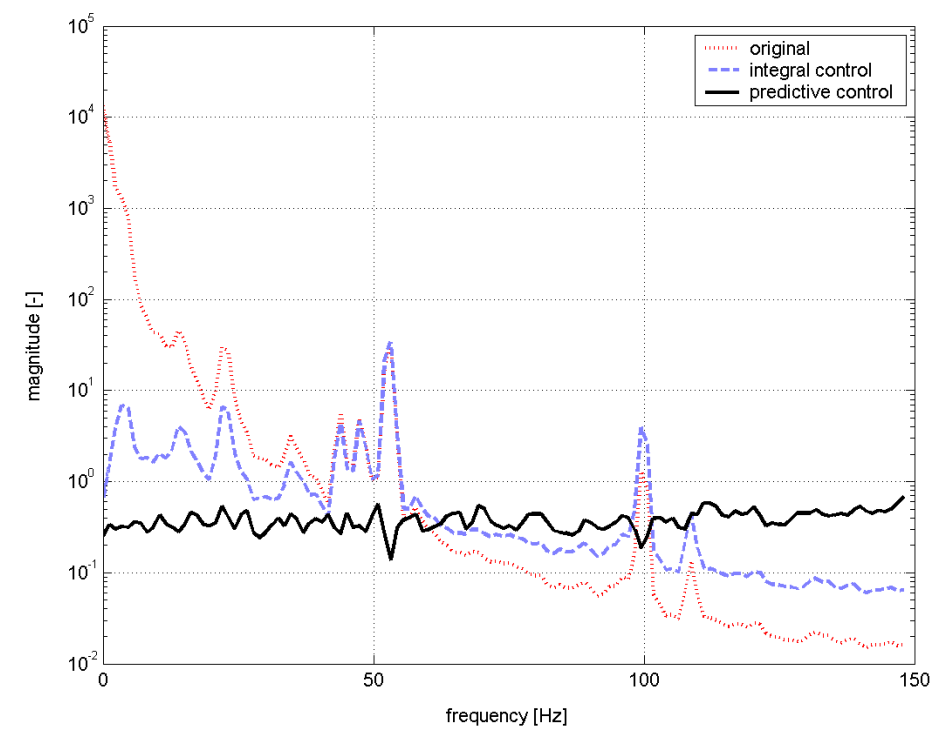

Fig. 8. Average power spectral density of the spatial aberration for the original phase estimate, integral control result and the predictive control result. The time delay is one sample instant.

The approach of including reference phase histories from neighbouring subapertures has also been applied to the JOSE datasets. However, the reduction that could be achieved on top of the purely temporal phase point prediction is marginal. This is an indication that Taylor's frozen flow hypothesis does not hold for these uncontrolled telescope data. It could be due to the fact that the wavefront distortion is the cumulative result of several atmospheric disturbance layers travelling with different velocities in different directions over the telescope aperture. The next section discusses the results obtained in a conditioned environment where the frozen flow hypothesis is valid.

\section{RESULTS ON AO TEST BENCH DATA}

At TNO TPD an AO test bench has been realised with an in-house built turbulent atmosphere emulator ${ }^{14}$. The emulator consists of a circular plan parallel glass plate mounted on a motor drive unit. Distortions are etched on one side of the glass plate such that it resembles a Kolmogorov spatial frequency distribution with a $D / r_{0}=5$, where $D$ is the diameter of the telescope and $r_{0}$ is Fried's parameter. In this way, a true single frozen layer disturbance can be emulated. The other main components of the test bench are a laser source, a Shack-Hartmann sensor, a CCD camera and a data acquisition system.

Uncontrolled Shack-Hartmann gradient data have been obtained with this test bench and phase estimates are calculated through a regularized Hudgin reconstruction. Purely temporal prediction and temporal prediction including information of one or two upstream neighbouring phase points has been compared to the integral control approach for different values of the Greenwood frequency; see Fig. 9. 
The results clearly show the advantage of including wavefront phase references from neighbouring subapertures for prediction. For low Greenwood frequencies of about $1 \mathrm{~Hz}(\mathbf{A})$ there is almost no difference in including information from neighbouring phase estimates compared to purely temporal prediction only. At a Greenwood frequency of $8 \mathrm{~Hz}(\mathbf{B})$ the system delay equals the time for the phase screen to travel over one subaperture distance. This finds expression in the fact that including one direct neighbour reference in the prediction shows a large performance gain. A similar effect can be distinguished at a Greenwood frequency of about $16 \mathrm{~Hz}$, where including the second neighbour shows the largest increase in performance compared to including only one direct neighbour $(\mathbf{C})$.

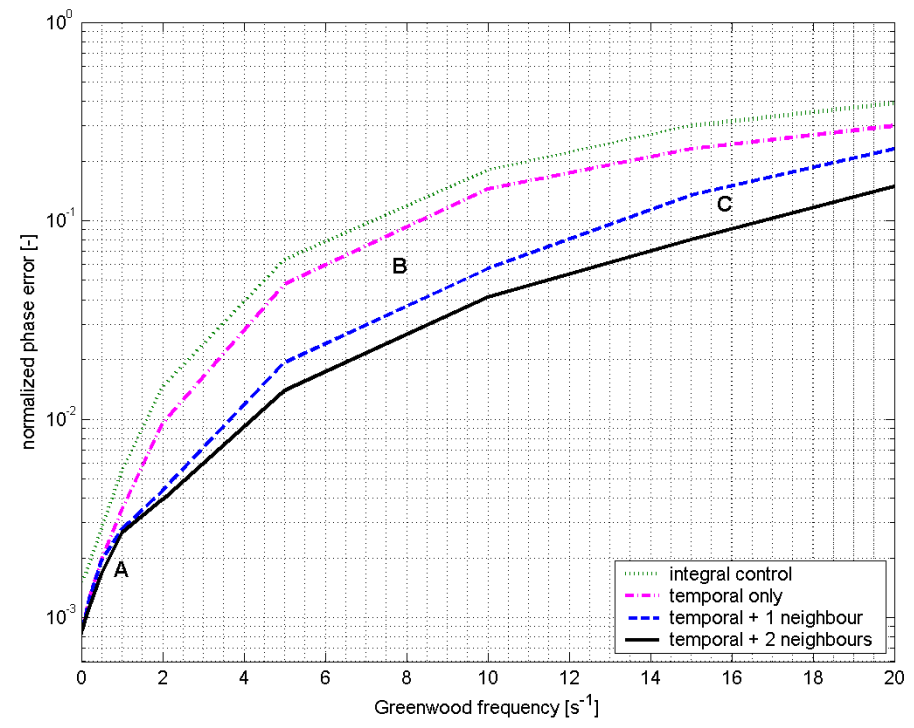

Fig. 9. The reduction of the mean squared error over time and space compared to the original open-loop test bench phase estimate data with a single layer frozen atmosphere disturbance as function of the Greenwood frequency.

This result clearly shows that for situations where Taylor's frozen layer assumption is valid, adding information of neighbouring upstream phase points has a positive effect on the quality of the prediction and hence the image quality that can be achieved. This is a promising technique for Multi Conjugate Adaptive Optics where atmospheric disturbance layers can be observed individually.

\section{CONCLUSIONS AND FUTURE OUTLOOK}

Analysing an AO system from a control point of view has led to the proposal of optimal control to reject the wavefront disturbance. The term 'optimal' refers to aiming at the minimum time- and space-averaged squared wavefront error. The AO controller can be regarded as a multiple-channel feedback regulator system, thus operating in closed-loop with the task to reject disturbances. This view differs from the common idea of having a feedforward servo command system.

Commonly, the controller is implemented as an integral controller. In the presence of a distinct time delay, a Smith predictor can be added to compensate for the closed-loop effects of the time delay. This approach can be denoted as heuristic. An optimal controller is very different in the sense that it is designed taking explicit account of the dynamics of the plant and the disturbance.

The design of the feedback optimal controller can be made more transparent by applying the concept of Internal Model Control. This yields an equivalent feedforward control structure. Turbulence prediction turns out to be one of the key elements of optimal control of the temporal wavefront error.

The potential of optimal control is demonstrated in 3 types of tests. From these tests it can be concluded that:

- optimal turbulence prediction may achieve a significant improvement of disturbance rejection compared to the approach of integral control with a Smith predictor.

- both the time history of a subaperture itself and time histories from neighbouring subaperture may function as valuable references for disturbance prediction. 
- time histories from subapertures in the upstream wind direction are especially useful in the case that the frozen flow hypothesis holds.

- for open-loop data from a single-conjugate AO system a clear flow direction can usually not be distinguished. In that case the best results are obtained with individual subaperture reference signals only.

A better rejection of the temporal error in AO system directly leads to a higher Strehl ratio. Alternatively, it gives the opportunity to have longer integration times in the wavefront sensor. This would reduce the wavefront measurement error, specifically useful in the case of a faint natural guide star.

Generally, an optimal control algorithm will demand more computational effort than an integral control algorithm. This could be a drawback for application in large-scale AO systems. Research effort needs to be spent to develop efficient implementation forms.

Our research on advanced control for AO systems in the forthcoming time will focus on:

- Further testing of the optimal control approach on more real telescope data. As a matter of fact inputs from observatories are welcome.

- Adapting the approach to multi-conjugate AO and testing it.

- Finalisation of the development of fast algorithms to realise optimal control on-line.

\section{ACKNOWLEDGMENTS}

We would like to thank the Cavendish astrophysics group of Cambridge University, UK, who designed and constructed the JOSE monitor, Richard Wilson of Durham University, UK, who provided the open-loop telescope data, and the Isaac Newton Group that operates the William Herschel Telescope.

The research presented here has been conducted in the framework of the "Knowledge Center for Aperture Synthesis". The knowledge center is an initiative of TNO TPD, to develop fundamental and advanced technologies for optical aperture synthesis. The knowledge center is a long-term co-operation of TNO and primarily the Delft University of Technology.

\section{REFERENCES}

1. J.W. Hardy, “Adaptive Optics for Astronomical Telescopes", Oxford University Press, New York, 1998.

2. M.A. van Dam, D. Le Mignaut and B.A. Macintosh, "Performance of the Keck Observatory adaptive optics system", submitted to Applied Optics, 2004.

3. G.C. Goodwin, S.F. Graebe and M.E. Salgado, "Control System Design", Prentice Hall, New Jersey, 2001.

4. K.J. Åström, "Limitations on Control System Performance", European Journal of Control 6, pp. 2-20, 2000.

5. Presentations at the IPAM Workshop on "Estimation and Control Problems in Adaptive Optics", UCLA, 2004.

6. E.P. Wallner, "Optimal wavefront correction using slope measurements", Journal of Opt. Soc. Am. 73, pp. 17711776, 1983.

7. W.J. Wild, "Lyapunov stability criteria for zonal adaptive optics systems", Optics Letters 23, pp.570-572, 1998.

8. P.Y. Madec, "Control techniques", Chapter 6 in "Adaptive Optics in Astronomy," edited by F. Roddier, Cambridge University Press, 1998.

9. M. Morari and E. Zafiriou, "Robust Process Control", Prentice Hall, New Jersey, 1989.

10. P.C. Mcguire et al, "Linear Zonal Atmospheric Prediction for Adaptive Optics", SPIE Conference on Adaptive Optics Systems and Technology, Vol. 4007, pp. 682-691, 2000.

11. C. Dessenne et al., "Optimization of a predictive controller for closed-loop adaptive optics", Applied Optics 37, pp. $4623-4633,1998$

12. K.A. Page and M. Shöck, "Analysis of atmospheric turbulence with applications to linear predictions", Proceedings of the ESO Conference "Beyond Conventional Adaptive Optics", Venice, 2001.

13. D. Saint-Jacques, "Astronomical Seeing in Space and Time", PhD thesis, University of Cambridge, 1998.

14. H. de Man, N. Doelman, M. Krutzen, "First Results with an Adaptive Optics Test Bench", Proceedings of SPIE Conference Adaptive Optical System Technologies II, Vol. 4839, pp. 121-130, 2002. 